\title{
Timosaponin A-III induces autophagy of T-cell acute lymphoblastic leukemia Jurkat cells via inhibition of the PI3K/Akt/mTOR pathway
}

\author{
HONG WANG $^{1 *}$, RUI DONG ${ }^{2 *}$, WEN-WEN FAN ${ }^{1}$, XING-CHANG ZHENG ${ }^{1}$, \\ AI-MIN LI ${ }^{3}$ and WEN-DI WANG ${ }^{1}$ \\ ${ }^{1}$ Department of Pediatrics, Qingdao Women and Children's Hospital; ${ }^{2}$ Department of Anesthesia Operation, \\ Qingdao Municipal Hospital, Qingdao University, Qingdao, Shandong 266000; \\ ${ }^{3}$ Department of Pediatrics, Yantai Yu Huang Ding Hospital, Qingdao University, \\ Yantai, Shandong 264003, P.R. China
}

Received June 29, 2018; Accepted February 14, 2019

DOI: $10.3892 /$ or.2019.7072

\begin{abstract}
Timosaponin A-III (TAIII) is a saponin isolated from anemarrhena asphodeloides and possesses the inhibitory effect on proliferation of multiple tumor cells. In the present study, the antitumor effect of TAIII and its underlying molecular mechanisms were investigated in vitro in T-cell acute lymphoblastic leukemia (T-ALL) Jurkat cells. The results demonstrated that TAIII inhibits the viability of Jurkat cells in a time- and dose-dependent manner, and induces apoptosis of Jurkat cells in a dose-dependent manner. Transmission electron microscopy demonstrated the formation of numerous autophagosomes in TAIII-treated Jurkat cells. Furthermore, monodansylcadaverine (MDC)-labeled autophagic vacuoles were observed following TAIII treatment by an inverted fluorescence microscope and MDC accumulation increased notably in TAIII treatment groups in a concentration-dependent manner. B-cell lymphoma-2 (Bcl-2)-associated X (Bax) was upregulated while Bcl-2 was reduced following TAIII treatment, indicating that the pro-apoptotic mechanism of TAIII may be associated with upregulation of Bax. Further investigation revealed that TAIII promotes the expression of autophagy-associated proteins Beclin 1 and LC3-II, and inhibits the phosphoinositide 3-kinase/Akt/mechanistic target of rapamycin kinase pathway. The present study revealed that the antitumor activity of TAIII was primarily achieved by the
\end{abstract}

Correspondence to: Professor Wen-Di Wang, Department of Pediatrics, Qingdao Women and Children's Hospital, Qingdao University, 217 West Liaoyang Road, Qingdao, Shandong 266000, P.R. China

E-mail:wwdmona1025@126.com

${ }^{*}$ Contributed equally

Key words: Timosaponin A-III, apoptosis, autophagy, T-cell acute lymphoblastic leukemia, mechanism induction of cell apoptosis and autophagy, indicating a promising potential as a novel effective reagent against T-ALL.

\section{Introduction}

Acute lymphoblastic leukemia (ALL) is the most common malignancy in childhood and accounts for $\sim 25 \%$ of cases diagnosed in children $<15$ years old (1). ALL is a heterogeneous disease that results from the malignant transformation of lymphoid progenitor cells in the bone marrow, blood and extramedullary sites (2). In children with ALL, T-cell ALL (T-ALL) accounts for 12-15\%, and compared with B-cell ALL, the prognosis of T-ALL is poorer due to increased relapse risk and reduced response to salvage therapy (3). In recent years, the outcome of children with T-ALL has improved with contemporary risk and response-based treatment protocols; additionally, the 5-year event-free survival rate reported by a number of foreign research centers reached $85 \%$, but the majority of the research centers remain at $\sim 60 \%$ (4-6). Currently, the primary focus for first-line therapy is on reducing the burden of long-term toxicity, decreasing the chemotherapy intensity for specific low-risk patients and strengthening treatment for high-risk groups (7). Since antineoplastic agents derived from natural products exhibit notable advantages, including low toxicity, insignificant or no side-effects, targeting multiple targets and a reduced chance to generate drug resistance (8), researchers focus on searching for novel antineoplastic drugs from natural products and Chinese traditional herbs.

Timosaponin A-III (TAIII), a steroidal saponin isolated from the rhizomes of anemarrhena asphodeloides, has been credited with a wide spectrum of bioactivities, including controlling hyperglycemia (9), improving learning and memory (10), inhibiting inflammation (11), suppressing allergic reaction (12) and restraining platelet aggregator (13). In recent years, more importance has been attached to its antitumor effect; additionally, accumulating evidence indicate that TAIII serves important roles in suppressing proliferation, inducing apoptosis $(14)$, activating autophagy $(15,16)$ and reversing multidrug resistant (17) of tumor cells. TAIII was observed to 
exhibit cytotoxicity towards a panel of carcinoma cell lines, including human breast carcinoma cells (MDA-MB-231 and MCF-7), hepatocellular carcinoma cells (Hep3b and HepG2), human lung cancer cells (A549), human colorectal cancer cells (HCT-15, HCT-116, HT-29, SW-480 and SW-620), human cervical epithelioid carcinoma cells (HeLa), human nasopharyngeal carcinoma cells (SUNE-1) and human chronic myeloid leukemia cell line (K562/ADM), but had a reduced effect on the viability of normal cells (16-19). However, to the best of our knowledge, limited research has been conducted to investigate the function of TAIII in T-ALL Jurkat cells.

In the present study, the aim was to investigate the effect of TAIII on cell apoptosis and autophagy in T-ALL Jurkat cells. It was determined that TAIII induced cell apoptosis and autophagy in a dose-dependent manner. Further study revealed that TAIII promoted autophagy via inhibiting the phosphoinositide 3-kinase (PI3K)/Akt/mechanistic target of rapamycin kinase (mTOR) pathway. The present study revealed the possible antitumor mechanisms of TAIII in T-ALL, providing novel strategies and targets for T-ALL treatment.

\section{Materials and methods}

Cell line and cell culture. The Human T-ALL Jurkat cell line was purchased from the Department of Pharmacology, The Institute of Hematology of Chinese Academy of Medical Sciences (Tianjin, China). The cells were maintained in RPMI-1640 medium supplemented with $10 \%$ fetal bovine serum (FBS; both from HyClone; GE Healthcare Life Sciences, Logan, UT, USA), $100 \mathrm{U} / \mathrm{ml}$ penicillin and $0.1 \mathrm{mg} / \mathrm{ml}$ streptomycin at $37^{\circ} \mathrm{C}$ in a humidified atmosphere containing $5 \%$ $\mathrm{CO}_{2}$. The cells cultured to $70-80 \%$ confluence were used in experiments.

Chemicals and reagents. TAIII (purity $>98 \%$; Shanghai Yuanye Bio-Technology Co., Ltd., Shanghai, China) and monodansylcadaverine (MDC; Sigma-Aldrich; Merck KGaA, Darmstadt, Germany) were dissolved in dimethyl sulfoxide (DMSO; Sigma-Aldrich; Merck KGaA) at the final concentration of $20 \mathrm{mM}$ and $50 \mu \mathrm{M}$, respectively, stored at $-20^{\circ} \mathrm{C}$ in the dark, and diluted in RPMI-1640 medium to the desired concentration prior to each experiment. The final DMSO concentration did not exceed $0.1 \%$ throughout the study. Rabbit polyclonal antibodies, including B-cell lymphoma 2 (Bcl-2; cat. no. bs-0032R), Bcl-2-associated X (Bax; cat. no. bs-20386R), Beclin 1 (cat. no. bs-1353R), LC3-I/LC3-II (cat. no. bs-2912R), Akt (cat. no. bs-0115R), phospho(p)-Akt (cat. no. bs-0876R), mTOR (cat. no. bs-1992R), p-mTOR (cat. no. bs-3495R), PI3K (cat. no. bs-6423R) and p-PI3K (cat. no. bs-4160R), were purchased from Beijing Biosynthesis Biotechnology Co., Ltd. (Beijing, China). Rabbit polyclonal antibody against GAPDH (cat. no. AB-P-R001) was a product of Hangzhou Goodhere Biotechnology Co., Ltd. (Hangzhou, China).

Cell Counting Kit-8 (CCK-8) assay for cell proliferation. CCK-8 (Dojindo Molecular Technologies, Inc., Shanghai, China) was used to determine the survival rate of cells incubated with TAIII. Jurkat cells were seeded into a 96-well plate at a density of $1 \times 10^{4}$ cells/well in RPMI-1640 containing $10 \%$
FBS. Subsequently, various concentrations of TAIII $(2,4,8,16$ and $32 \mu \mathrm{M}$ ) were added. After the cells were incubated at $37^{\circ} \mathrm{C}$ in an atmosphere containing $5 \% \mathrm{CO}_{2}$ for 24,48 or $72 \mathrm{~h}, 10 \mu \mathrm{l}$ CCK-8 solution was added to each well and incubated for an additional $4 \mathrm{~h}$ at $37^{\circ} \mathrm{C}$. The absorbance was measured at $580 \mathrm{~nm}$ with Model 680 microplate reader (Bio-Rad Laboratories, Inc., Hercules, CA, USA). A blank well containing only RPMI-1640 medium supplemented with $10 \%$ FBS was used as a control.

Cell apoptosis analysis by flow cytometry. Jurkat cells were plated into a 6 -well plate at a concentration of $3 \times 10^{5}$ cells in $1 \mathrm{ml} \mathrm{RPMI-1640} \mathrm{medium} \mathrm{supplemented} \mathrm{with} \mathrm{10 \%} \mathrm{FBS.}$ Following incubation with TAIII $(0,2$ and $8 \mu \mathrm{M})$ at $37^{\circ} \mathrm{C}$ for $24 \mathrm{~h}$, the cells were harvested and washed twice with ice-cold PBS. Subsequently, the cells were suspended in suspended in $400 \mu \mathrm{l} 1 \mathrm{X}$ binding buffer (Nanjing KeyGen Biotech Co., Ltd., Nanjing, China) and double-stained with Annexin V-fluorescein isothiocyanate/propidium iodide (Annexin V-FITC/PI; Nanjing KeyGen Biotech Co., Ltd.) for $15 \mathrm{~min}$ in the dark at room temperature. The apoptosis cells were analyzed using a FACSCanto ${ }^{\mathrm{TM}}$ II Flow Cytometer with CellQuest software V 7.5.3 (BD Biosciences; Becton, Dickinson and Company, Franklin Lakes, NJ, USA). The apoptotic rate was calculated as the percentage of early apoptotic cells plus the percentage of late apoptotic cells.

Observation of cell ultrastructure under transmission electron microscopy (TEM). Jurkat cells were incubated with TAIII ( 0 and $8 \mu \mathrm{M})$ at $37^{\circ} \mathrm{C}$ for $24 \mathrm{~h}$. Subsequently, cells were harvested, washed twice with PBS, fixed with $3 \%$ glutaraldehyde for $24 \mathrm{~h}$ at $4^{\circ} \mathrm{C}$. Following washing three times with ice-cold PBS, Jurkat cells were progressively dehydrated in $30 \%$ ethanol for 5-10 $\mathrm{min}, 50 \%$ ethanol for 5-10 min, $70 \%$ ethanol for 5-10 $\mathrm{min}, 90 \%$ ethanol for $10-15 \mathrm{~min}, 100 \%$ ethanol for 10-15 min and embedded in Epon 812 resin. Finally, the specimens were sliced into serial ultrathin sections (thickness, $100 \mathrm{~nm}$ ), and stained in uranyl acetate for 15-30 min and lead citrate for 5-10 $\mathrm{min}$ at room temperature. The specific ultrastructure features were observed under a TEM (JEM-2800; JEOL, Ltd., Tokyo, Japan; x4,000 magnification).

Visualization of MDC-labeled autophagic vacuoles. MDC staining of autophagic vacuoles was performed for autophagy analysis (20). Jurkat cells growing on coverslips were pretreated with TAIII $(0,2$ and $8 \mu \mathrm{M})$ for $24 \mathrm{~h}$ at $37^{\circ} \mathrm{C}$. Following treatments, the cells were stained with $50 \mu \mathrm{M}$ MDC in PBS for $10 \mathrm{~min}$ at $37^{\circ} \mathrm{C}$, and then washed three times with PBS to remove excess MDC and subsequently analyzed under an inverted fluorescence microscopy (F-7000; Hitachi, Ltd., Tokyo, Japan; x400 magnification). Fluorescence of MDC was measured at $365 \mathrm{~nm}$ excitation filter, $395 \mathrm{~nm}$ spectroscope and $420 \mathrm{~nm}$ absorption filter.

Detection of intracellular mean fluorescence intensity of $M D C$. Jurkat cells were plated into a 6 -well plate at a concentration of $3 \times 10^{5}$ cells in $1 \mathrm{ml}$ RPMI-1640 medium supplemented with $10 \% \mathrm{FBS}$. The cells were pretreated with TAIII $(0,2$ and $8 \mu \mathrm{M})$ at $37^{\circ} \mathrm{C}$ for $24 \mathrm{~h}$. Subsequently, the cells were harvested by centrifugation at $3,000 \mathrm{x}$ g for $3 \mathrm{~min}$ at room temperature and suspended with $1 \mathrm{ml}$ RPMI-1640 medium 
supplemented with $10 \%$ FBS. Following this, MDC (50 $\mu \mathrm{M})$ was added to each group and dyed for $10 \mathrm{~min}$ in the dark at $37^{\circ} \mathrm{C}$. After staining, the cells were collected by centrifugation at 3,000 $\mathrm{x}$ g for $3 \mathrm{~min}$ at room temperature, washed twice with ice-cold PBS and suspended with $1 \mathrm{ml}$ ice-cold PBS. Immediately after that, the cell-associated mean fluorescence intensity (MFI) was detected at $488 \mathrm{~nm}$ excitation wavelength by a FACSCanto ${ }^{\mathrm{TM}}$ II Flow Cytometer with CellQuest software V 7.5.3.

Western blot assay. The cells were plated into a 6-well plate at a concentration of $3 \times 10^{5}$ and cultured to $70-80 \%$ confluence at $37^{\circ} \mathrm{C}$ for $24 \mathrm{~h}$. The cells were harvested and washed with PBS twice, and then scraped and lysed in Radioimmunoprecipitation Assay buffer (Beyotime Institute of Biotechnology, Shanghai, China). Protein concentrations were determined by Bicinchoninic Acid Protein Assay kit (Beyotime Institute of Biotechnology). Protein samples $(40 \mu \mathrm{g})$, including Beclin 1 (50 kDa), Akt (56 kDa), p-Akt (56 kDa), mTOR (289 kDa), p-mTOR (289 kDa), PI3K (82 kDa), p-PI3K (82 kDa) and GAPDH $(37 \mathrm{kDa})$, were separated with $8 \%$ SDS-PAGE (Beyotime Institute of Biotechnology). Bcl-2 (26 kDa), Bax (21 kDa) and LC3-I/LC3-II (14/16 kDa) were separated with $15 \%$ SDS-PAGE. Subsequently, the proteins were transferred onto polyvinylidene difluoride membranes (EMD Millipore, Billerica, MA, USA). The membranes were blocked by $5 \%$ skimmed dry milk in TBS containing $0.2 \%$ Tween 20 at room temperature for $2 \mathrm{~h}$ followed by an overnight incubation with specific antibodies at $4^{\circ} \mathrm{C}$. The primary antibodies were rabbit polyclonal antibodies against Bcl-2 (1:500), Bax (1:500), Beclin 1 (1:500), LC3-I/LC3-II (1:500), Akt (1:500), p-Akt (1:500), mTOR (1:500), p-mTOR (1:500), PI3K (1:500), p-PI3K (1:500) and GAPDH (1:1,000). Following three washes in TBS/Tween buffer, the membranes were incubated in horseradish peroxidase-labeled goat anti-rabbit immunoglobulin $\mathrm{G}$ (1:5,000; cat. no. TA130015; OriGene Technologies, Inc., Beijing, China) for $2 \mathrm{~h}$ at $37^{\circ} \mathrm{C}$. Detection was performed using the FluorChem FC2 gel imaging system (ProteinSimple, San Jose, CA, USA). Each band density was quantified using Image J V 1.8.0 image processing program (National Institutes of Health, Bethesda, MD, USA) and normalized by GAPDH for their respective lanes.

Data analysis. Statistical analyses were conducted using SPSS 21.0 software (IBM Corp., Armonk, NY, USA). The normality of data was analyzed by the Shapiro-Wilk test, and it was determined that the data were normally distributed. One-way analysis of variance was used to analyze differences between $\geq 3$ groups, followed by a Least Significant Difference multiple range test for post-hoc comparisons. Data are expressed as the means \pm standard deviations. $\mathrm{P}<0.05$ was considered to indicate a statistically significant difference.

\section{Results}

TAIII suppresses proliferation of Jurkat cells. The CCK-8 assay was conducted to evaluate the cytotoxicity of TAIII on the proliferation of Jurkat cells. As depicted in Fig. 1, Jurkat cells were sensitive to TAIII and TAIII inhibited the viability of Jurkat cells in a time- and dose-dependent manner in vitro.

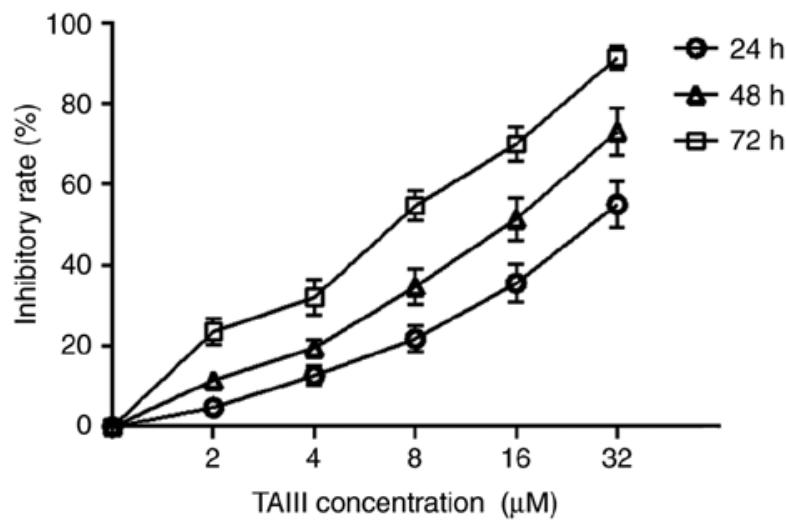

Figure 1. TAIII inhibits the proliferation of Jurkat cells. Jurkat cells were treated with $2,4,8,16$ or $32 \mu \mathrm{M}$ TAIII for 24,48 or $72 \mathrm{~h}$. Growth curves were based on data from Cell Counting Kit- 8 assays. The results are represented as the mean \pm standard deviation of triplicate experiments. TAIII, Timosaponin A-III.

The inhibitory rate of TAIII on growth of Jurkat cells was $4.34 \pm 0.31,13.67 \pm 0.78$ and $22.15 \pm 1.04 \%$, respectively, after the cells were treated with $2 \mu \mathrm{M}$ TAIII for 24,48 and $72 \mathrm{~h}$.

TAIII induces apoptosis of Jurkat cells. As depicted in Fig. 2A-C, after treatment with 0,2 or $8 \mu \mathrm{M}$ TAIII for $24 \mathrm{~h}$, the total apoptosis rate of Jurkat cells was $4.63 \pm 1.47$, $27.07 \pm 2.57$ and $55.13 \pm 5.17 \%$, respectively. Additionally, TAIII induced apoptosis of Jurkat cells in a dose-dependent manner (Fig. 2D).

Autophagy detection by TEM. TEM was performed to detect the micro-morphological change of Jurkat cells. Results demonstrated that TAIII induces Jurkat cells to generate autophagy. As depicted in Fig. 3, Jurkat cells not treated with TAIII exhibited the normal ultrastructural morphology of nuclei, cytoplasm and organelles. The most prominent morphological change in TAIII-treated cells was the formation of numerous autophagic vacuoles in the cytoplasm, and the giant cytophagosomes filled with degraded organelles and autolysosomes were frequently observed.

Observation of vacuolization in cytoplasm by inverted fluorescence microscope. MDC-labeled autophagic vacuoles were observed by inverted fluorescence microscope. As depicted in Fig. 4, MDC-labeled autophagic vacuoles appeared as distinct dot-like structures distributing in cytoplasm or in perinuclear regions. The TAIII-treated group exhibited increased fluorescent density and more MDC-labeled particles, compared with the control group, indicating that TAIII induced the formation of the MDC-labeled vacuoles. Additionally, it was determined that vacuolization in the cytoplasm progressively became larger and denser when the concentration of TAIII increased.

MDC accumulation increases in Jurkat cells following TAIII treatment. Furthermore, flow cytometry was used to detect intracellular MDC MFI. The results implied that the MFI of MDC in TAIII-treated Jurkat cells increased in a dose-dependent manner, compared with untreated Jurkat cells. As depicted in Fig. 5, Jurkat cells treated with TAIII $(2$ and $8 \mu \mathrm{M})$ 
A

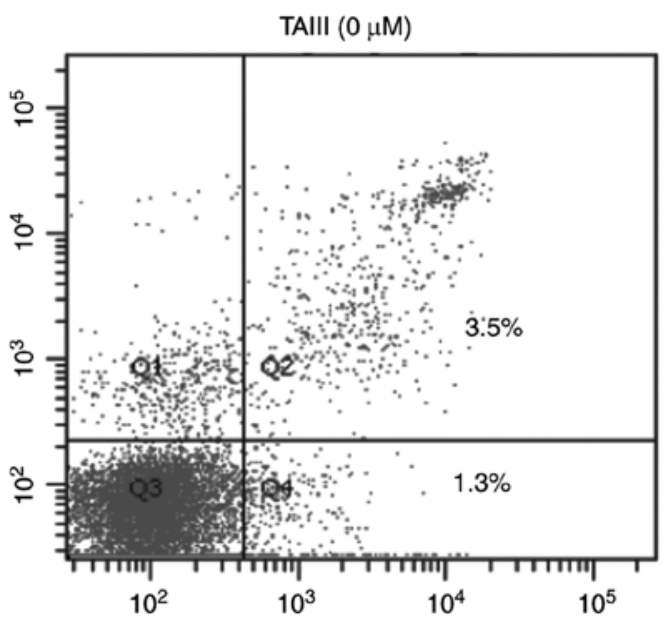

C

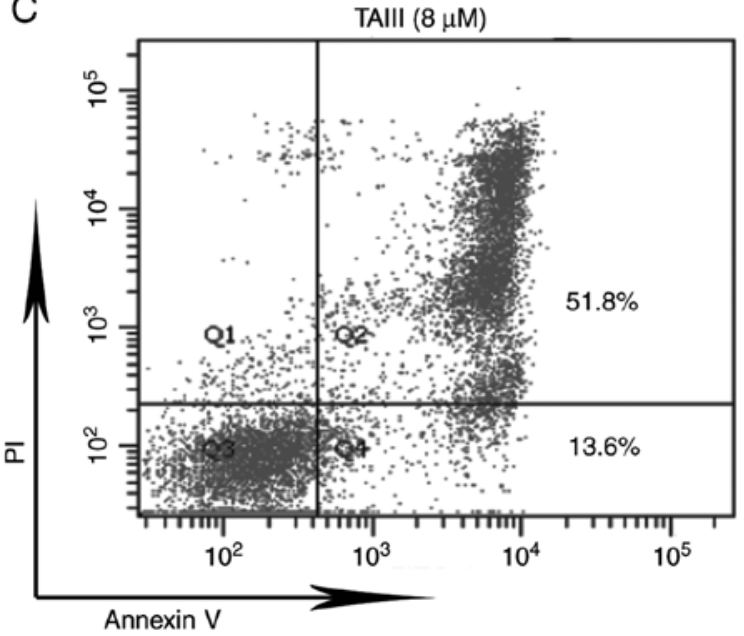

B

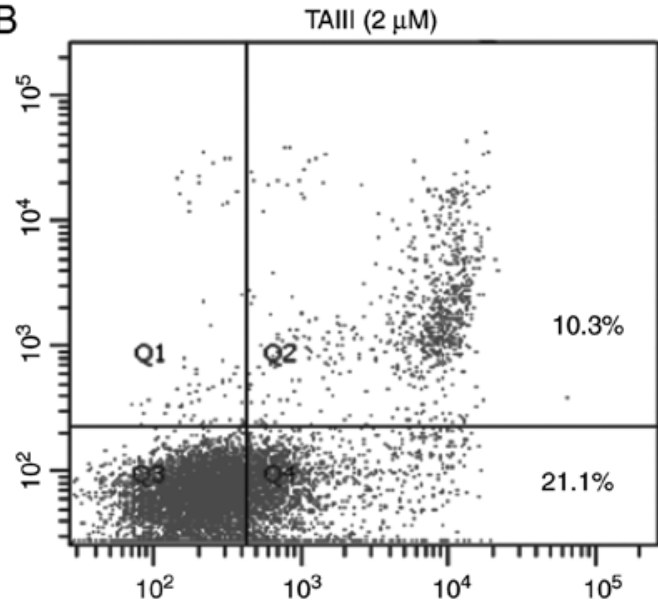

D

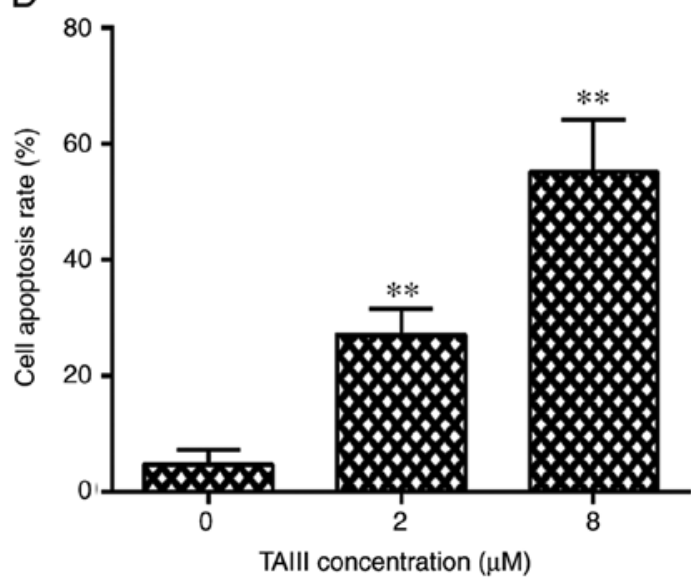

Figure 2. TAIII induces apoptosis of Jurkat cells. Annexin V-fluorescein isothiocyanate/PI staining was conducted in order to measure cell apoptosis. (A) Jurkat cells treated with RPMI-1640. (B) Jurkat cells treated with $2 \mu$ M TAIII. (C) Jurkat cells treated with $8 \mu$ M TAIII. (D) A graph representing the analysis of cell apoptotic ratio of three groups. Data are expressed as mean \pm standard deviation of triplicate experiments. ${ }^{* *} \mathrm{P}<0.01$ vs. $0 \mu \mathrm{M}$ TAIII. TAIII, Timosaponin A-III; PI, propidium iodide.

A

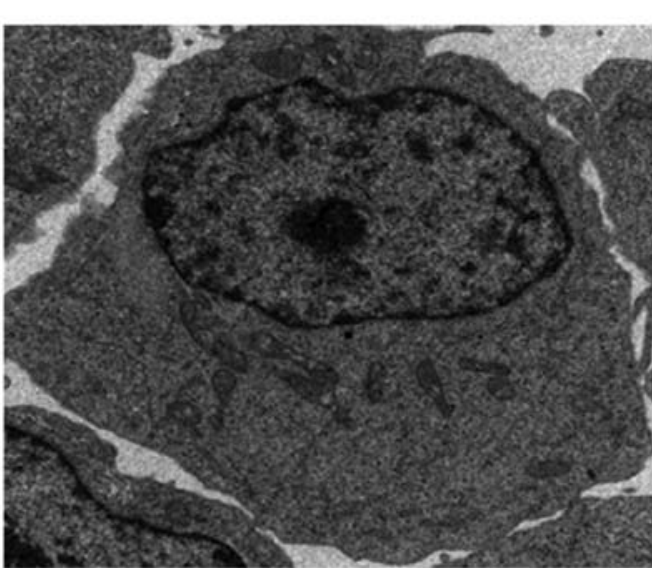

TAIII $(0 \mu \mathrm{M})$
B

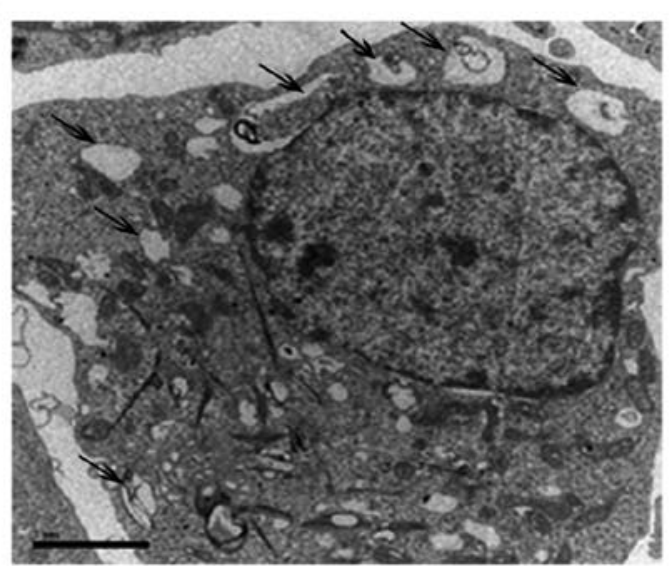

TAIII $(8 \mu \mathrm{M})$

Figure 3. Transmission electron microscopy depicts the formation of autophagosomes in TAIII-treated Jurkat cells. (A) Jurkat cell not treated with TAIII revealed normal morphology of nuclei, cytoplasm and cell organelles. (B) Jurkat cell treated with TAIII exhibit the formation of numerous autophagic vacuoles. x4,000 magnification. Black arrows represent autophagosomes. TAIII, Timosaponin A-III.

enhanced the MDC MFI by 1.31- and 1.76-fold, compared with the control group.
TAIII increases Bax and decreases Bcl-2 expression in Jurkat cells. To elucidate the molecular mechanism underlying the 


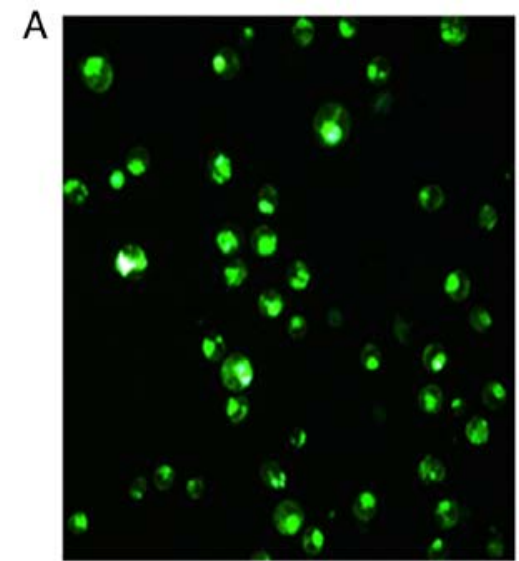

TAIII $(0 \mu \mathrm{M})$

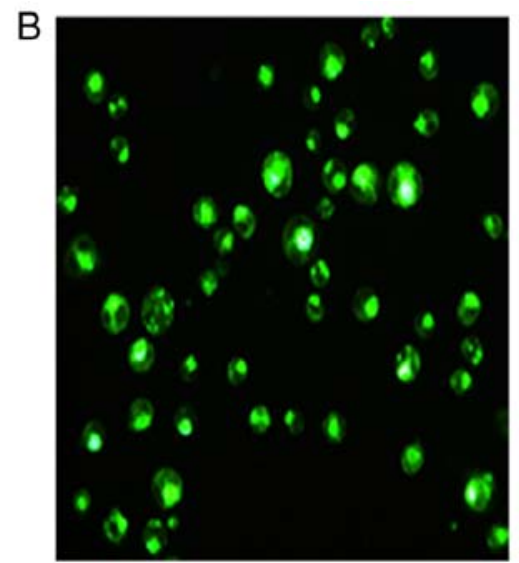

TAIII $(2 \mu \mathrm{M})$

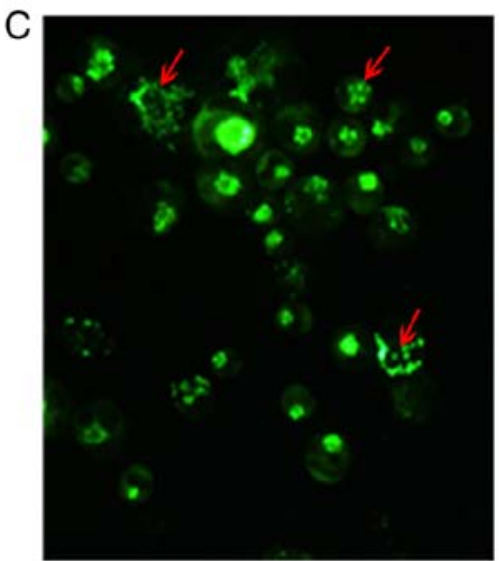

TAIII $(8 \mu \mathrm{M})$

Figure 4. Inverted fluorescence microscopy depicts the formation of autophagic vacuoles by staining with MDC in TAIII-treated Jurkat cells. (A) Jurkat cells treated with RPMI-1640. (B) Jurkat cells treated with $2 \mu$ M TAIII. (C) Jurkat cells treated with $8 \mu$ M TAIII. x400 magnification. Red arrows represent autophagic vacuoles. TAIII, Timosaponin A-III.
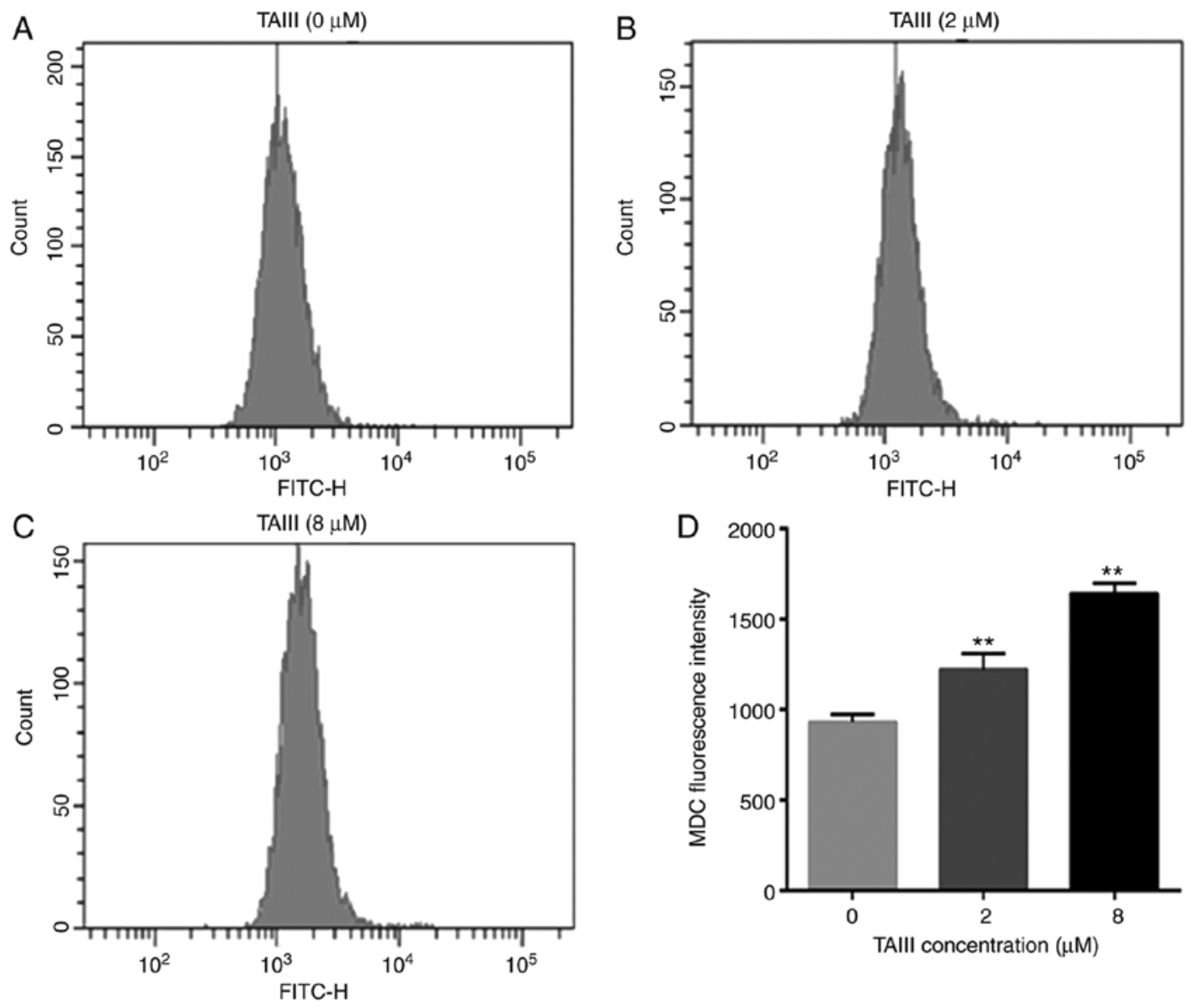

Figure 5. TAIII increases the intracellular accumulation of MDC. (A) Jurkat cells treated with RPMI-1640. (B) Jurkat cells treated with $2 \mu$ M TAIII. (C) Jurkat cells treated with $8 \mu \mathrm{M}$ TAIII. (D) A graph representing the analysis of intracellular MDC mean fluorescence intensity. Data are expressed as mean \pm standard deviation values of triplicate experiments. ${ }^{* *} \mathrm{P}<0.01$ vs. $0 \mu \mathrm{M}$ TAIII. TAIII, Timosaponin A-III; MDC, monodansylcadaverine.

apoptosis induced by TAIII, the protein expression levels of Bcl-2 and Bax genes, apoptosis-associated molecules, were evaluated by western blot assay. The results demonstrated that the expression of Bax increases significantly while the expression of Bcl-2 decreases after Jurkat cells incubation with different concentrations of TAIII for $24 \mathrm{~h}$ (Fig. 6).
TAIII upregulates Beclin 1 and LC3-II expression in Jurkat cells. To clarify the mechanism underlying the autophagy induced by TAIII, western blotting was conducted to assess the effect of TAIII on the expression of Beclin 1 and LC3-II, which serve key roles in autophagy $(18,20)$. As depicted in Fig. 7A and B, TAIII treatment caused the conversion of 
A
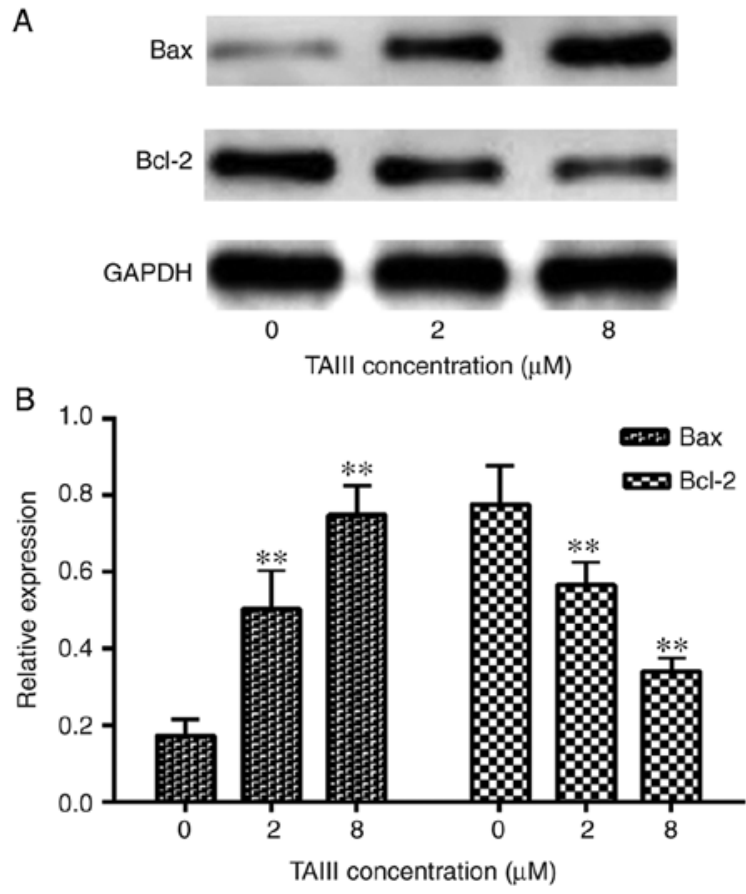

Figure 6. Effect of TAIII treatment on expression of Bcl-2 and Bax in Jurkat cells. TAIII upregulated the protein level of Bax and downregulated the protein level of Bcl-2 in Jurkat cells. (A) Western blot analysis. (B) Analysis of expression of Bcl-2 and Bax proteins. The results are represented as the mean \pm standard deviation of triplicate experiments. ${ }^{* *} \mathrm{P}<0.01$ vs. $0 \mu \mathrm{M}$ TAIII. TAIII, Timosaponin A-III; Bcl-2, B-cell lymphoma-2; Bax, Bcl-2-associated X.

LC3 from the cytoplasmic form (LC3-I) into the autophagosomic form (LC3-II), while also increasing the expression of Beclin 1, in Jurkat cells in a concentration-dependent manner.

TAIII promotes Jurkat cells autophagy via PI3K/Akt/mTOR signaling. It is known that the PI3K/Akt/mTOR signaling pathway serves a suppressive role in autophagy (21). In the present study, the activity of the PI3K/Akt/mTOR signaling pathway was investigated by TAIII treatment in Jurkat cells. As depicted in Fig. 7C and D, western blotting demonstrated that the levels of p-PI3K, p-Akt and p-mTOR are notably reduced in TAIII treatment groups, compared with the control group. However, there were no notable changes in the total amount of PI3K, Akt and mTOR. These results indicated that PI3K/Akt/mTOR signaling is involved in Jurkat cells autophagy induced by TAIII.

\section{Discussion}

TAIII, one of the main active constituents of Chinese medicinal herb anemarrhena asphodeloides bunge, has been credited with effective anticancer activities, including suppressing proliferation, enhancing apoptosis, activating autophagy and reversing multidrug resistance $(14,16,17)$. Sy et al $(16)$ reported that TAIII-induced autophagy in human cervical HeLa cells, followed by mitochondria-dependent apoptotic cell death. It was demonstrated that TAIII reverses multi-drug resistance in human chronic myelogenous leukemia K562/ADM cells via downregulation of MDR1 and MRP1 expression via inhibiting the PI3K/Akt signaling pathway (17). Kang et al (18) determined that TAIII inhibits the proliferation of human colon cancer HCT-15 cells with cell cycle arrest and induction of apoptosis. In the present study, results indicated that TAIII exerts a significant inhibitory effect on Jurkat cells in a time- and dose-dependent manner, and induces tumor cell apoptosis via downregulating Bcl-2 expression. Additionally, Jurkat cells autophagy was promoted by the inhibition $\mathrm{PI} 3 \mathrm{~K} / \mathrm{Akt} / \mathrm{mTOR}$ signaling. Overall, the results demonstrated that TAIII stimulates cell apoptosis and autophagy of Jurkat cells in vitro.

Considering that apoptosis induction is one of the key features of antitumor drugs in tumor therapy, it is important to examine the effects of TAIII on apoptosis induction and tumor growth. Apoptosis, also known as type I programmed cell death, is characterized by morphological changes, including cell shrinkage, chromatin condensation and membrane bleeding, without disruption of the plasma membrane; additionally, apoptosis is triggered by two pathways, the death receptor-mediated extrinsic pathway and the mitochondrial-involved intrinsic pathway (21-24). Bcl-2 family members are critical players in mitochondrial-involved intrinsic apoptosis (25). Bax and Bcl-2 are the most characterized apoptosis regulators in mitochondrial-associated apoptosis; furthermore, Bax is the first identified pro-apoptotic protein member of the Bcl-2 protein family, possessing promoting apoptosis activity (26). In the present study, data demonstrated that TAIII induces apoptosis of Jurkat cells in a dose-dependent manner (Fig. 2). However, consistent with the ability of TAIII to kill cells via apoptosis processes, TAIII increased the expression of Bax and decreased the expression of Bcl-2 in a dose-dependent manner (Fig. 6), indicating that the upregulated Bax and downregulated Bcl-2 expression may trigger TAIII-induced apoptosis of Jurkat cells.

Furthermore, for the first time, the present study also provides experimental evidence to demonstrate the activation of an autophagic program in Jurkat cells following treatment with TAIII. Autophagy is a genetically programmed and evolutionarily conserved process, and macro-, micro-, and chaperone-mediated autophagy are three primary autophagy forms, with macro-autophagy being the most prevalent form (27). Autophagy can regulate physiological and pathophysiological cell death, and the basic function of autophagy is to sustain survival and maintain cell homeostasis through organelles and proteins recycling $(28,29)$. Increasing evidence indicate that autophagy is important in human cancer suppression and extensive attention has been paid to its role in tumor therapy $(30,31)$. Previous studies indicated that TAIII is a pronounced activator of autophagy $(15,16,32)$. In the present study, TEM determined the formation of autophagosomes, which are the representative characteristics of autophagy (Fig. 3). Furthermore, numerous MDC-labeled autophagic vacuoles were observed in TAIII-treated Jurkat cells under an inverted fluorescence microscope (Fig. 4), and flow cytometry analysis illustrated that the MDC accumulation increased notably in TAIII treatment groups in a dose-dependent manner (Fig. 5).

In order to further examine the mechanism by which TAIII regulated autophagy, various molecular studies were performed. Beclin 1, a mammalian orthologue of the yeast Apg6/Vps30 gene and a subunit of the class III PI3-kinase complex, is the first mammalian gene that was identified to be 

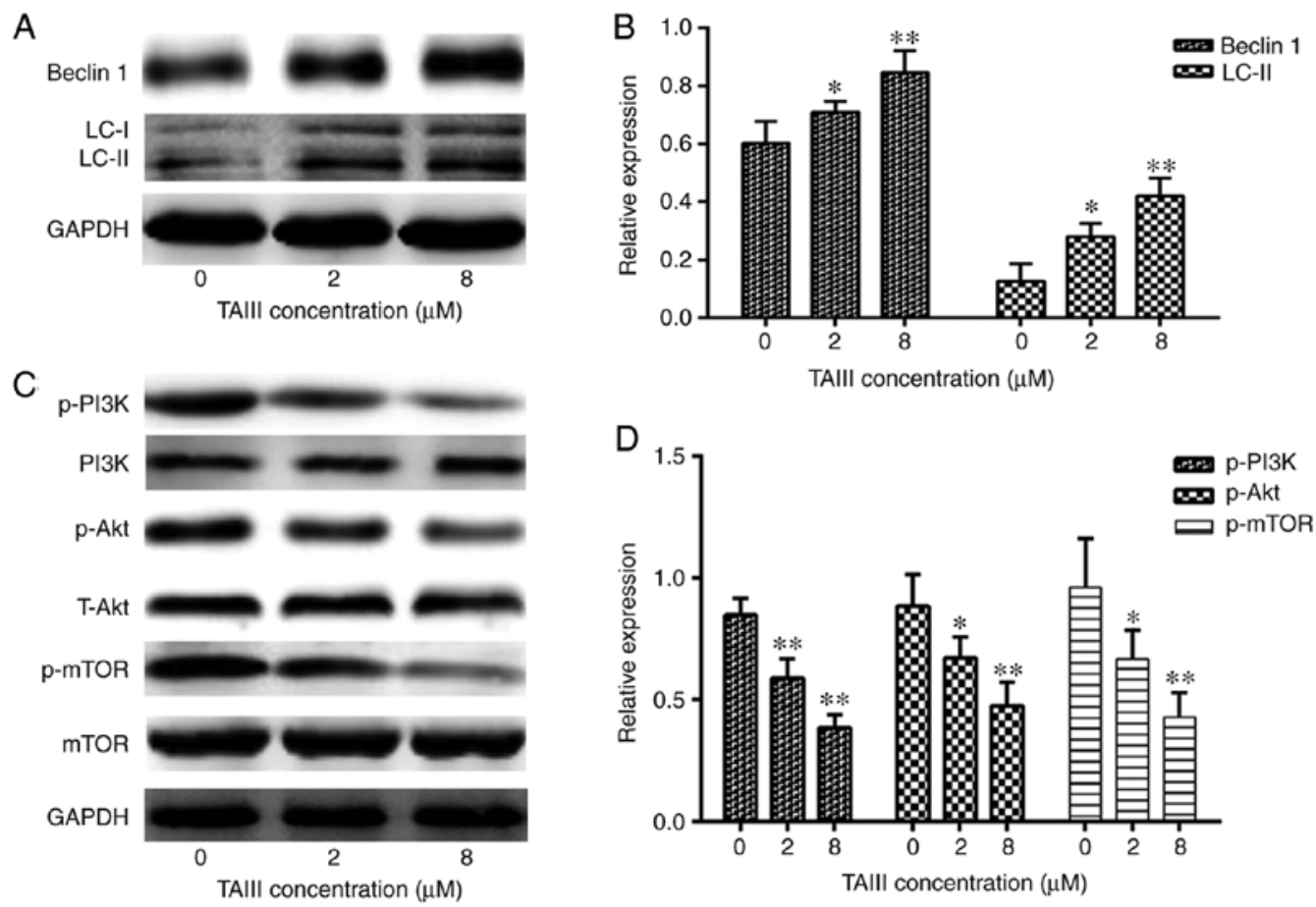

Figure 7. TAIII promotes Beclin 1 and LC3-II expression via PI3K/Akt/mTOR signaling in Jurkat cells. Jurkat cells were treated with TAIII at different concentrations $(0,2$ and $8 \mu \mathrm{M})$ for $24 \mathrm{~h}$ and the expression of Beclin 1, LC3-II, p-PI3K, p-AKT and p-mTOR was analyzed by western blot analysis. (A) Western blot analysis of Beclin 1 and LC3-II. (B) Analysis of Beclin 1 and LC3-II expression. (C) Western blot analysis of PI3K, p-PI3K, T-Akt, p-Akt, mTOR and p-mTOR. (D) Analysis of p-PI3K, p-Akt and p-mTOR expression. All results are expressed as the mean \pm standard deviation of triplicate experiments. ${ }^{*} \mathrm{P}<0.05$ and ${ }^{* *} \mathrm{P}<0.01$ vs. $0 \mu \mathrm{M}$ TAIII. TAIII, Timosaponin A-III; PI3K, phosphoinositide 3-kinase; mTOR, mechanistic target of rapamycin kinase; p-, phospho-.

able to induce autophagy (33). LC3-II has been identified as a marker for autophagosome formation (8). Therefore, the expression levels of Beclin 1 and LC3-II in TAIII-treated Jurkat cells were measured. As depicted in Fig. 7, TAIII treatment upregulated the expression of Beclin 1 and LC3-II in Jurkat cells in a concentration-dependent manner. The results demonstrated that TAIII induces the expression of the autophagy-associated proteins Beclin 1 and LC3-II, while reducing the proliferation of Jurkat cells in vitro. There have been a substantial amount of studies indicating that PI3K/Akt/mTOR signaling are key axes involved in anticancer drug-induced autophagy $(8,34,35)$. Further investigation was performed, with a conclusion that the PI3K/Akt/mTOR signaling pathway was inactivated by TAIII through suppressing the expression of p-PI3K, p-Akt and p-mTOR. While the preliminary observations are promising, more comprehensive and detailed studies are required to be conducted, for example, animal models in vivo or more cellular experiments, including a colony form assay, and other apoptosis indicators, including a DNA fragmentation assay.

In conclusion, the present results demonstrate that TAIII possesses antitumor activity and its antineoplastic action is associated with inhibition of proliferation, and induction of apoptosis and autophagy in Jurkat cells. Furthermore, the results demonstrated that the anticancer activity of TAIII in Jurkat cells may be through regulating apoptosis-associated proteins, including Bax and Bcl-2, and autophagy-associated proteins, including Beclin 1 and LC3-II. Furthermore, TAIII promoted Jurkat cells autophagy through the PI3K/Akt/mTOR pathway. Finally, the present data may provide a novel approach for the development of T-ALL therapy.

\section{Acknowledgements}

Not applicable.

\section{Funding}

The present study was supported by Shandong Province medical science and technology development project (grant no. 2016WS0701), the Science and Technology Benefiting Project of Qingdao City (15-9-2-83-nsh), the Science and Technology Project of Yantai (grant no. 2016WS003), and the Scientific researching fund projects of Yantai Yu Huang Ding Hospital (grant no. 201504).

\section{Availability of data and materials}

The datasets analyzed during the present study are available from the corresponding author upon reasonable request.

\section{Authors' contributions}

HW performed the experiments, analyzed the results and wrote the manuscript. RD and WWF were responsible for the collection and assembly of data, and the data analysis. XCZ and AML revised the manuscript and performed supplementary experiments. WDW was responsible for design of the present study and financial support. All authors read and approved the manuscript and agree to be accountable for all aspects of the research in ensuring that the accuracy or integrity of any part of the work are appropriately investigated and resolved. 


\section{Ethics approval and consent to participate}

Not applicable.

\section{Patient consent for publication}

Not applicable.

\section{Competing interests}

The authors declare that they have no competing interests.

\section{References}

1. Bassan R, Bourquin JP, DeAngelo DJ and Chiaretti S: New approaches to the management of adult acute lymphoblastic leukemia. J Clin Oncol: Sep 21, 2018 (Epub ahead of print). JCO2017773648. doi: 10.1200/JCO.2017.77.3648.

2. Brassesco MS, Pezuk JA, Cortez MA, Bezerra Salomão K, Scrideli CA and Tone LG: TLE1 as an indicator of adverse prognosis in pediatric acute lymphoblastic leukemia. Leuk Res 74: 42-46, 2018

3. Patrick K and Vora A: Update on biology and treatment of T-cell acute lymphoblastic leukaemia. Curr Opin Pediatr 27: 44-9, 2015

4. Matloub Y, Stork L, Asselin B, Hunger SP, Borowitz M, Jones T, Bostrom B, Gastier-Foster JM, Heerema NA, Carroll A, et al Outcome of children with standard-risk T-lineage acute lymphoblastic leukemia - Comparison among different treatment strategies. Pediatr Blood Cancer 63: 255-261, 2016.

5. Gaynon PS, Angiolillo AL, Carroll WL, Nachman JB, Trigg ME, Sather HN, Hunger SP and Devidas M: Long-term results of the children's cancer group studies for childhood acute lymphoblastic leukemia 1983-2002: A Children's Oncology Group Report. Leukemia 24: 285-297, 2010

6. Silverman LB, Stevenson KE, O'Brien JE, Asselin BL, Barr RD, Clavell L, Cole PD, Kelly KM, Laverdiere C, Michon B, et al: Long-term results of Dana-Farber Cancer Institute ALL Consortium protocols for children with newly diagnosed acute lymphoblastic leukemia (1985-2000). Leukemia 24: 320-334, 2010 .

7. Huang W, Wang WT, Fang K, Chen ZH, Sun YM, Han C, Sun LY, Luo XQ and Chen YQ: MIR-708 promotes phagocytosis to eradicate T-ALL cells by targeting CD47. Mol Cancer 17: 12, 2018.

8. You P, Wu H, Deng M, Peng J, Li F and Yang Y: Brevilin A induces apoptosis and autophagy of colon adenocarcinoma cell CT26 via mitochondrial pathway and PI3K/AKT/mTOR inactivation. Biomed Pharmacother 98: 619-625, 2018.

9. Tang YH, Sun ZL, Fan MS, Li ZX and Huang CG: Anti-diabetic effects of TongGuanWan, a Chinese traditional herbal formula, in C57BL/KsJ-db/db mice. Planta Med 78: 18-23, 2012.

10. Lee B, Jung K and Kim DH: Timosaponin AIII, a saponin isolated from Anemarrhena asphodeloides, ameliorates learning and memory deficits in mice. Pharmacol Biochem Behav 93: 121-127, 2009

11. Lim SM, Jeong JJ, Kang GD, Kim KA, Choi HS and Kim DH: Timosaponin AIII and its metabolite sarsasapogenin ameliorate colitis in mice by inhibiting $\mathrm{NF}-\kappa \mathrm{B}$ and MAPK activation and restoringTh17/Treg cell balance. Int Immunopharmacol 25 : 493-503, 2015 .

12. Lee B, Trinh HT, Jung K, Han SJ and Kim DH: Inhibitory effects of steroidal timosaponins isolated from the rhizomes of Anemarrhena asphodeloides against passive cutaneous anaphylaxis reaction and pruritus. Immunopharmacol Immunotoxicol 32: 357-363, 2010.

13. Wang GJ, Lin LC, Chen CF, Cheng JS, Lo YK, Chou KJ, Lee KC, Liu CP, Wu YY Su W, et al: Effect of Timosaponin A-III, from Anemarrhenae asphodeloides Bunge (Liliaceae), on calcium mobilization in vascular endothelial and smooth muscle cells and on vascular tension. Life Sci 71: 1081-1090, 2002.

14. Huang HL, Chiang WL, Hsiao PC, Chien MH, Chen HY Weng WC, Hsieh MJ and Yang SF: Timosaponin AIII mediates caspase activation and induces apoptosis through JNK1/2 pathway in human promyelocytic leukemia cells. Tumour Biol 36: 3489-3497, 2015.
15. Lok CN, Sy LK, Liu F and Che CM: Activation of autophagy of aggregation- prone ubiquitinated proteins by timosaponin A-III. J Biol Chem 286: 31684-31696, 2011.

16. Sy LK, Yan SC,Lok CN, Man RY and Che CM: Timosaponin A-III induces autophagy preceding mitochondria-mediated apoptosis in HeLa cancer cells. Cancer Res 68: 10229-10237, 2008.

17. Chen JR, Jia XH, Wang H, Yi YJ, Wang JY and Li YJ: Timosaponin A-III reverses multi-drug resistance in human chronic myelogenous leukemia K562/ADM cells via downregulation of MDR1 and MRP1 expression by inhibiting PI3K/Akt signaling pathway. Int J Oncol 48: 2063-2070, 2016.

18. Kang YJ, Chung HJ, Nam JW, Park HJ, Seo EK, Kim YS, Lee D and Lee SK: Cytotoxic and antineoplastic activity of timosaponin A-III for human colon cancer cells. J Nat Prod 74: 701-706, 2011.

19. Nho KJ, Chun JM and Kim HK: Induction of mitochondria-dependent apoptosis in HepG2 human hepatocellular carcinoma cells by timosaponin A-III. Environ Toxicol Pharmacol 45: 295-301, 2016.

20. Biederbick A, Kern HF and Elsasser HP: Monodansylcadaverine (MDC) is a specific in vivo marker for autophagic vacuoles. Eur J Cell Biol 66: 3-14, 1995.

21. Kwak CH, Lee SH, Lee SK, Ha SH, Suh SJ, Kwon KM, Chung TW, Ha KT, Chang YC, Lee YC, et al: Induction of apoptosis and antitumor activity of eel skin mucus, containing lactose-binding molecules, on human leukemic K562 cells. Mar Drugs 13: 3936-3949, 2015.

22. Scarfò L and Ghia P: Reprogramming cell death: BCL2 family inhibition in hematological malignancies. Immunol Lett 155 : 36-39, 2013

23. Qi L, Ren K, Fang F, Zhao DH, Yang NJ and Li Y: Over expression of BCL2 and low expression of caspase 8 related to TRAIL resistance in brain cancer stem cells. Asian Pac J Cancer Prev 16: 4849-4852, 2015.

24. Christodoulou MI, Kontos CK, Halabalaki M, Skaltsounis AL and Scorilas A: Nature promises new anticancer agents: Interplay with the apoptosis-related BCL2 gene family. Anticancer Agents Med Chem 14: 375-399, 2014

25. Wang S,Zhou M,Ouyang J, Geng Z and Wang Z: Tetraarsenictetrasulfide and arsenic trioxide exert synergistic effects on induction of apoptosis and differentiation in acute promyelocytic leukemia cells. PLoS One 10: e0130343, 2015.

26. Cingeetham A, Vuree S, Dunna NR, Gorre M, Nanchari SR, Edathara PM, Meka P, Annamaneni S, Digumarthi R, Sinha S and Satti V: Influence of BCL2-938C $>$ A and BAX-248G $>$ A promoter polymorphisms in the development of AML: Case-control study from South India. Tumour Biol 36: 7967-7976, 2015.

27. Glick D, Barth S and Macleod KF: Autophagy: Cellular and molecular mechanisms. J Pathol 221: 3-12, 2010.

28. Wilde L, Tanson K, Curry J and Martinez-Outschoorn U: Autophagy in cancer: A complex relationship. Biochem J 475: 1939-1954, 2018.

29. Mizushima N: The pleiotropic role of autophagy: from protein metabolism to bactericide. Cell Death Differ 12 (Suppl 2): S1535-S1541, 2005.

30. Altman BJ, Jacobs SR, Mason EF, Michalek RD, MacIntyre AN, Coloff JL, Ilkayeva O, Jia W, He YW and Rathmell JC: Autophagy is essential to suppress cell stress and to allow BCR-Abl-mediated leukemogenesis. Oncogene 30: 1855-1867, 2011.

31. Gong A, Ye S, Xiong E, Guo W, Zhang Y, Peng W, Shao G, Jin J, Zhang Z, Yang J, et al: Autophagy contributes to ING4-induced glioma cell death. Exp Cell Res 319: 1714-1723, 2013.

32. Wang N, Feng Y, Zhu M, Siu FM, Ng KM and Che CM: A novel mechanism of XIAP degradation induced by Timosaponin AIII in hepatocellular carcinoma. Biochim Biophys Acta 1833: 2890-2899, 2013.

33. Qu X, Yu J, Bhagat G, Furuya N, Hibshoosh H, Troxel A, Rosen J, Eskelinen EL, Mizushima N, Ohsumi Y, et al: Promotion of tumorigenesis by heterozygous disruption of the beclin 1 autophagy gene. J Clin Invest 112: 1809-20, 2003.

34. Xu Y, Cai X, Zong B, Feng R, Ji Y, Chen G and Li Z: Qianlie Xiaozheng decoction induces autophagy in human prostate cancer cells via inhibition of the Akt/mTOR pathway. Front Pharmacol 9: 234, 2018.

35. Lin H, Zhang C, Zhang H, Xia YZ, Zhang CY, Luo J, Yang L and Kong LY: Physakengose G induces apoptosis via EGFR/mTOR signaling and inhibits autophagic flux in human osteosarcoma cells. Phytomedicine 42: 190-198, 2018. 